IJMMS 25:1 (2001) 1-10

PII. S0161171201004495

http://ijmms.hindawi.com

(C) Hindawi Publishing Corp.

\title{
ON THE EXISTENCE OF SOLUTIONS FOR A CLASS OF FIRST-ORDER DIFFERENTIAL EQUATIONS
}

\author{
KAMEL AL-KHALED
}

(Received 22 November 1999 and in revised form 10 March 2000)

\begin{abstract}
A system of first-order differential equations with linear constraint is studied. Existence theorems for the solution are proved under some conditions. Some uniqueness and dependence results for the system are also obtained. Some applications are given.
\end{abstract}

2000 Mathematics Subject Classification. Primary 34G10, 34B05, 34C27.

1. Introduction. Let $\mathbb{R}^{n}$ denote Euclidean $n$-space. If $x=\left[x_{i}\right]=\left(x_{1}, x_{2}, \ldots, x_{n}\right)^{T}$ denotes an element of $\mathbb{R}^{n}$, let

$$
\|x\| \equiv \sum_{i=1}^{n}\left|x_{i}\right| .
$$

If $B=\left[b_{i j}\right]$ is an $n \times n$ matrix over $\mathbb{R}$, let $\|B\|$ denote the corresponding matrix norm, that is,

$$
\|B\| \equiv \sup _{\|x\|=1}\|B x\| .
$$

The Banach space of continuous functions $x(t)$ mapping $[a, b]$ into $\mathbb{R}^{n}$ under the norm

$$
\|x\| \equiv \max _{[a, b]}\|x(t)\|
$$

will be denoted by $C[a, b]$. If $B(t)=\left[b_{i j}(t)\right]$ is a continuous matrix function on $[a, b]$, we define

$$
\|B\| \equiv \max _{[a, b]}\|B(t)\| .
$$

This paper is concerned with problems which consist of an ordinary differential equation or system of equations together with one or more linear side conditions. The most general problem considered is, for $r \in \mathbb{R}^{n}$,

$$
x^{\prime}=A(t) x+f(t, x)
$$

together with the linear constraint

$$
\mathbf{L} x=r .
$$

We will assume throughout this paper the following assumptions:

(A1) $A(t)$ is a continuous $n \times n$ matrix function on $[a, b]$.

(A2) $f(t, x)$ is continuous on $\mathbf{D}=\mathbf{D}(a, b)=\left\{(t, x): t \in[a, b], x \in \mathbb{R}^{n}\right\}$ with values in $\mathbb{R}^{n}$. 
(A3) $\mathbf{L}=\mathbf{L}(a, b)$ is a bounded, linear mapping from $C[a, b]$ into $\mathbb{R}^{n}$ with bound $\|\mathbf{L}\| \equiv \sup _{\|x\|=1}\|\mathbf{L} x\|$.

By a solution to (1.5) on $[a, b]$ we mean a function $x(t) \in C[a, b]$ which has a continuous derivative on $[a, b]$ and satisfies (1.5) on $[a, b]$.

Many other authors have studied under some conditions the existence and uniqueness of solutions for systems of first-order differential equations. For example, Heikkilä [6] derive existence and comparison results for extremal solutions of a firstorder ordinary differential equation in an ordered Banach space. Bobisud and O'Regan [2] consider the initial value problem for a first-order differential equation $y^{\prime}=F(t, y)$ and consider hypotheses which ensure the uniqueness for a two-point boundary value problem. While in [4], representation and approximation of the solutions to the linear evolution equation

$$
x^{\prime}(t)+A x(t)=g(t)
$$

are studied. For more research papers, see [7, 8].

In this paper, we use the Schauder fixed point theorem [3] to develop an existence theory for problem (1.5), (1.6). Though the basic existence result, Theorem 3.1, is close to a theorem proved by Antosiewicz [1], numerous variations and consequences are obtained which do not appear in the literature.

2. Preliminary results. We prove a sequence of lemmas which are needed in order to prove our main result. To illustrate the general results obtained for the problem in (1.5), (1.6), we from time to time consider the following particular mappings from $C[a, b]$ into $\mathbb{R}^{n}$ :

$$
\begin{aligned}
& \mathbf{L}_{0} x \equiv x(a), \\
& \mathbf{L}_{1} x \equiv\left(x_{1}\left(t_{1}\right), x_{2}\left(t_{2}\right), \ldots, x_{n}\left(t_{n}\right)\right), \quad t_{i} \in[a, b], \\
& \mathbf{L}_{2} x \equiv \int_{a}^{b} x(s) d s .
\end{aligned}
$$

LEMMA 2.1. The mappings $\mathbf{L}_{0}, \mathbf{L}_{1}$, and $\mathbf{L}_{2}$ satisfy (A3), that is, $\mathbf{L}_{0}, \mathbf{L}_{1}, \mathbf{L}_{2}$ are bounded, linear mappings from $C[a, b]$ into $\mathbb{R}^{n}$. In particular, $\left\|\mathbf{L}_{0}\right\|=1,\left\|\mathbf{L}_{1}\right\| \leq n$, and $\left\|\mathbf{L}_{2}\right\|=$ $b-a$.

Proof. The linearity of $\mathbf{L}_{0}, \mathbf{L}_{1}$, and $\mathbf{L}_{2}$ is immediate. We have

$$
\left\|\mathbf{L}_{0}(x)\right\|=\|x(a)\| \leq \max _{[a, b]}\|x(t)\|=\|x\| ;
$$

with equality for $x(t) \equiv c \in \mathbb{R}^{n}$; that is, $\left\|\mathbf{L}_{0}\right\|=1$. We have

$$
\left\|\mathbf{L}_{1}(x)\right\|=\sum_{i=1}^{n}\left|x_{i}\left(t_{i}\right)\right| \leq n\|x\| ;
$$

that is, $\left\|\mathbf{L}_{1}\right\| \leq n$. We have

$$
\left\|\mathbf{L}_{2}(x)\right\|=\left\|\int_{a}^{b} x(s) d s\right\| \leq \int_{a}^{b}\|x(s)\| d s \leq(b-a)\|x\|
$$

with equality for $x(t) \equiv c \in \mathbb{R}^{n}$; that is, $\left\|\mathbf{L}_{2}\right\|=(b-a)$. 
2.1. A variation of the Schauder fixed point theorem. As a basic tool in determining sufficient conditions for existence of a solution to (1.5), (1.6) we will use the following fact by Schauder [3].

LEMMA 2.2. Let $K$ be a convex, compact subset of a normed linear space $X$, then any continuous mapping $T$ from $K$ into $K$ has a fixed point in $K$.

In order to obtain a more useful form of Lemma 2.2, we state the following lemma.

LEMMA 2.3 (see [3]). Let $X$ be a Banach space and $C \subset X$ be compact, then the closed convex hull of $C$ is compact.

LEMMA 2.4. Let $X$ be a Banach space and let $K$ be a closed convex subset of $X$. If $T$ is a continuous map of $K$ into itself such that $T(K)$ is relatively compact, then $T$ has a fixed point in $K$.

Proof. Since $T(K) \subset K$ and $K$ is closed we have $\overline{T(K)} \subset K$. Let $K^{0}$ denote the closed convex hull of $\overline{T(K)}$. By Lemma $2.3, K^{0}$ is compact. Since $K^{0}$ is the smallest closed convex set containing $\overline{T(K)}$, we must have $K^{0} \subset K$. Thus $T\left(K^{0}\right) \subset T(K) \subset \overline{T(K)} \subset K^{0}$. By Lemma 2.2, $T$ has a fixed point in $K^{0}$ which must also lie in $K$.

2.2. A general existence theorem. In addition to (A1), (A2), and (A3), we impose the following condition:

(A4) The linear problem

$$
x^{\prime}=A(t) x, \quad \mathbf{L} x=r,
$$

has a unique solution for any $r \in \mathbb{R}^{n}$; that is, if $S$ denotes the $n$-dimensional space of solutions to $x^{\prime}=A(t) x$, then $(\mathbf{L} \mid S)^{-1}$ exists.

REMARK 2.5. Property (A4) is equivalent to the condition that $z(t) \equiv 0$ be the only element in $S$ such that $\mathbf{L} z=0$; that is, the null space of $\mathbf{L} \mid S$ is $\{0\}$.

Condition (A4) together with the following lemmas will enable us to define an appropriate mapping for application of Lemma 2.4.

LEMMA 2.6. If $A(t)$ satisfies (A1), then the problem

$$
x^{\prime}=A(t) x+z(t), \quad x\left(t_{0}\right)=r .
$$

has a unique solution $\omega(t)$ on $[a, b)$ for any $t_{0} \in[a, b], z \in C[a, b]$, and $r \in \mathbb{R}^{n}$. Moreover, for any $t, t_{0}$ in $[a, b]$,

$$
\|\omega(t)\| \leq\left\|\omega\left(t_{0}\right)\right\| \exp \left|\int_{t_{0}}^{t}\|A(s)\| d s\right|+\left|\int_{t_{0}}^{t} \exp \right| \int_{\tau}^{t}\|A(s)\| d s|\|z(\tau)\| d \tau| .
$$

The existence and uniqueness of solutions to such initial value problems is well known (cf. [5]). The estimate may also be found in [5].

LEMMA 2.7. If (A1), (A3), and (A4) are satisfied, then the problem

$$
x^{\prime}=A(t) x+z(t), \quad \mathbf{L} x=r,
$$

has a unique solution $w(t)$ for any $r \in \mathbb{R}^{n}$ and $z \in C[a, b]$. 
Proof. Let

$$
w(t) \equiv \omega_{0}(t)+(\mathbf{L} \mid S)^{-1}\left(-\mathbf{L} \omega_{0}\right)+(\mathbf{L} \mid S)^{-1} r,
$$

where $\omega_{0}$ is the unique solution to

$$
x^{\prime}=A(t) x+z(t), \quad x(a)=0 .
$$

It is easily verified by differentiation that $w(t)$ is a solution to (2.8). If $w_{1}(t), w_{2}(t)$ are two solutions to (2.8), then $w_{1}(t)-w_{2}(t)$ is the unique solution to

$$
x^{\prime}=A(t) x, \quad L x=0 .
$$

Hence by property (A4), $w_{1}(t) \equiv w_{2}(t)$; that is, $w(t)$ is the unique solution to (2.8).

LEMMA 2.8. Suppose (A1) and (A4) are satisfied. If $\hat{w}_{0}(t)$ is the unique solution to (2.8) with $r=0$, then

$$
\left\|\hat{w}_{0}\right\| \leq \int_{a}^{b}\left(\exp \int_{\tau}^{b}\|A(s)\| d s\right)\|z(\tau)\| d \tau\left(1+\left\|(\mathbf{L} \mid S)^{-1} \mathbf{L}\right\|\right) .
$$

In particular,

$$
\left\|\hat{w}_{0}\right\| \leq K_{1}\|z\|
$$

where $K_{1}=(b-a) \exp \int_{a}^{b}\|A(s)\| d s\left(1+\left\|(\mathbf{L} \mid S)^{-1} \mathbf{L}\right\|\right)$.

Proof. We have from (2.9) that

$$
\hat{w}_{0}(t)=\omega_{0}(t)+(\mathbf{L} \mid S)^{-1}\left(-\mathbf{L} \omega_{0}\right),
$$

where $\omega_{0}(t)$ is the unique solution to the initial value problem

$$
x^{\prime}=A(t) x+z(t), \quad x(a)=0 .
$$

Thus

$$
\left\|\hat{w}_{0}\right\| \leq\left\|\omega_{0}\right\|+\left\|(\mathbf{L} \mid S)^{-1} \mathbf{L}\right\|\left\|\omega_{0}\right\| \leq\left\|\omega_{0}\right\|\left(1+\left\|(\mathbf{L} \mid S)^{-1} \mathbf{L}\right\|\right) .
$$

From Lemma 2.6 with $t_{0}=a$ and $r=0$, we have

$$
\left\|\omega_{0}\right\| \leq \int_{a}^{b}\left(\exp \int_{\tau}^{b}\|A(s)\| d s\right)\|z(\tau)\| d \tau
$$

and (2.12) follows.

The following notation will be used extensively in the remainder of this paper. Let $H$ be a positive number, $\vec{H}=\left(H_{1}, H_{2}, \ldots, H_{n}\right)$, where each $H_{i}$ is positive, and $H(t)$ be a continuous, positive function for $a \leq t \leq b$. Let

$$
\begin{aligned}
& \psi(r)=\psi(t ; r, a, b) \equiv(\mathbf{L}(a, b) \mid S)^{-1} r, \\
& C(H)=C(H, r, a, b) \equiv\{y \in C[a, b]:\|y-\psi(r)\| \leq H\}, \\
& D(H)=D(H, r, a, b) \equiv\{(t, y) \in D(a, b):\|y-\psi(t ; r)\| \leq H\}, \\
& D(H, t)=D(H, r, t) \equiv\left\{y \in \mathbb{R}^{n}:\|y-\psi(t ; r)\| \leq H\right\}, \\
& C(H(t))=C(H(t), r, a, b) \equiv\{y \in C[a, b]:\|y(t)-\psi(t ; r)\| \leq H(t), \forall t \in[a, b]\}, \\
& C(\vec{H})=C(\vec{H}, r, a, b) \equiv\left\{y \in C[a, b]:\left\|y_{i}(t)-\psi_{i}(t ; r)\right\| \leq H_{i}, i=1,2, \ldots, n ; t \in[a, b]\right\} .
\end{aligned}
$$


Note that $C(H), C(H(t))$, and $C(\vec{H})$ are closed, convex subsets of $C[a, b]$. Note also that if $y \in C(H)$, then $(t, y(t)) \in D(H)$ and $y(t) \in D(H, t)$ for $t \in[a, b]$.

If (A2) is satisfied and $y \in C[a, b]$, then $f(t, y(t))$ is continuous on $[a, b]$. By Lemma 2.7, the problem

$$
x^{\prime}=A(t) x+f(t, y(t)), \quad \mathbf{L} x=r,
$$

has a unique solution. We denote this solution by $u(r, y)=u(t ; r, y)$. Note that

$$
u(r, y)=\psi(r)+u(0, y)
$$

We now define a mapping $T$ on $C[a, b]$ by

$$
T(y) \equiv u(r, y)
$$

Note that if $T x=x$, then $x$ is a solution to the problem (1.5), (1.6).

LEMMA 2.9. If (A1) through (A4) are satisfied, then $T(C(H))$ is relatively compact in $C[a, b]$.

Proof. By Lemma 2.8 and (2.20), for $y \in C(H)$, we have

$$
\begin{aligned}
\|T y\| & =\|u(r, y)\| \leq\|\psi(r)\|+\|u(0, y)\| \\
& \leq\left\|(\mathbf{L} \mid S)^{-1}\right\|\|r\|+K_{1} \max _{[a, b]}\|f(t, y(t))\| \\
& \leq\left\|(\mathbf{L} \mid S)^{-1}\right\|\|r\|+K_{1} \max _{D(H)}\|f(t, z)\| \equiv B_{1} ;
\end{aligned}
$$

that is, $T(C(H))$ is bounded by $B_{1}$. By Ascoli's theorem, it is sufficient to show that $T(C(H))$ is equicontinuous. For $y \in C(H), T y=u(r, y)$ and

$$
\left\|u^{\prime}(t ; r, y)\right\|=\|A(t) u(t ; r, y)+f(t, y(t))\| \leq\|A\| B_{1}+\max _{D(H)}\|f(t, z)\| \equiv B_{2} .
$$

By the mean value theorem, for $t_{1}, t_{2} \in[a, b]$,

$$
\left\|u\left(t_{2} ; r, y\right)-u\left(t_{1} ; r, y\right)\right\| \leq B_{2}\left|t_{2}-t_{1}\right| .
$$

Thus $T(C(H))$ is equicontinuous.

LEMMA 2.10. If (A1) through (A4) are satisfied, then $T$ is continuous on $C(H)$.

Proof. Let $\epsilon>0$ be given. Since $D(H)$ is compact, $f$ is uniformly continuous on $D(H)$. There exists $\delta>0$ such that if $\left(t_{1}, x_{1}\right)$ and $\left(t_{2}, x_{2}\right)$ are in $D(H)$ and $\left|t_{1}-t_{2}\right|+$ $\left\|x_{1}-x_{2}\right\|<\delta$, then $\left\|f\left(t_{1}, x_{1}\right)-f\left(t_{2}, x_{2}\right)\right\|<\epsilon / K_{1}$, where $K_{1}$ is defined in Lemma 2.8.

If $y_{1}, y_{2} \in C(H)$, then $T y_{1}-T y_{2}$ is the solution to

$$
x^{\prime}=A(t) x+f\left(t, y_{1}(t)\right)-f\left(t, y_{2}(t)\right), \quad \mathbf{L} x=0 .
$$

By Lemma 2.8, we have

$$
\left\|T y_{1}-T y_{2}\right\| \leq K_{1} \max _{[a, b]}\left\|f\left(t, y_{1}(t)\right)-f\left(t, y_{2}(t)\right)\right\|
$$


If $\left\|y_{1}-y_{2}\right\|<\delta$; that is, $\left\|y_{1}(t)-y_{2}(t)\right\|<\delta$ for $t \in[a, b]$, then

$$
\max _{[a, b]}\left\|f\left(t, y_{1}(t)\right)-f\left(t, y_{2}(t)\right)\right\|<\frac{\epsilon}{K_{1}}, \quad\left\|T y_{1}-T y_{2}\right\|<K_{1} \frac{\epsilon}{K_{1}}=\epsilon .
$$

Hence, $T$ is continuous on $C(H)$.

3. Main results. With the aid of the preceding lemmas we can now prove our main results.

THEOREM 3.1. Suppose (A1) through (A4) are satisfied. If there exists $H>0$ such that

$$
M(H)=M(H, r, a, b) \equiv \sup _{y \in C(H)}\|u(0, y)\| \leq H,
$$

then problem (1.5), (1.6) has a solution $x(t) \in C(H)$.

Proof. From (2.20) we have $T y-\psi(r)=u(r, y)-\psi(r)=u(0, y)$. Thus, for $y \in C(H)$, (3.1) yields

$$
\|T y-\psi(r)\|=\|u(0, y)\| \leq H
$$

that is, $T y \in C(H)$, and $T(C(H)) \subset C(H)$. Since $C(H)$ is closed and convex, we can conclude from Lemmas 2.9 and 2.10 and the Schauder theorem in the form of Lemma 2.4 that $T$ has a fixed point $x \in C(H)$; that is, problem (1.5), (1.6) has a solution $x \in C(H)$.

We may easily obtain some natural generalization of Theorem 3.1.

THEOREM 3.2. Suppose (A1) through (A4) are satisfied. If there exists a positive, continuous function $H(t)$ on $[a, b]$ such that

$$
M(t, H(t)) \equiv \sup _{y \in C(H(t))}\|u(t ; 0, y)\| \leq H(t)
$$

for $t \in[a, b]$, then the problem (1.5), (1.6) has a solution $x \in C(H(t))$.

Proof. We have $T y(t)-\psi(t ; r)=u(t ; r, y)-\psi(t ; r)=u(t ; 0, y)$. The condition (3.3) implies that, for $y \in C(H(t))$,

$$
\|T y(t)-\psi(t ; r)\|=\|u(t ; 0, y)\| \leq H(t)
$$

for $t \in[a, b]$. Thus $T y \in C(H(t))$; that is, $T(C(H(t))) \subset C(H(t))$. If $H \equiv \max _{[a, b]} H(t)$, then $C(H(t)) \subset C(H)$. Moreover, $T(C(H(t))) \subset T(C(H))$. By Lemma 2.9, $T(C(H))$ is a relatively compact subset of $C[a, b]$; hence, $T(C(H(t)))$ is relatively compact. By Lemma 2.10, $T$ is continuous on $C(H)$; hence $T$ is continuous on $C(H(t))$. Since $C(H(t))$ is a closed, convex subset of $C[a, b]$, we may conclude from Lemma 2.4 that $T$ has a fixed point $x$ in $C(H(t))$; that is, problem (1.5), (1.6) has a solution $x \in C(H(t))$.

THEOREM 3.3. Suppose (A1) through (A4) are satisfied. If there exists $\vec{H}=$ $\left(H_{1}, H_{2}, \ldots, H_{n}\right)$ such that

$$
M_{i}(\vec{H})=\sup _{y \in C(\vec{H})}\left(\max _{[a, b]}\left|u_{i}(t ; 0, y)\right|\right) \leq H_{i}
$$

for $i=1,2, \ldots, n$, then problem (1.5), (1.6) has a solution $x \in C(\vec{H})$. 
Proof. From (2.20), we have

$$
(T y)_{i}(t)-\psi_{i}(t ; r)=u_{i}(t ; r, y)-\psi_{i}(t ; r)=u_{i}(t ; 0, y)
$$

for $i=1,2, \ldots, n$ and $t \in[a, b]$. Condition (3.5) implies that, for $y \in C(\vec{H})$,

$$
\left|(T y)_{i}(t)-\psi_{i}(t ; r)\right|=\left|u_{i}(t ; 0, y)-\psi_{i}(t ; r)\right|=\left|u_{i}(t ; 0, y)\right| \leq H_{i}
$$

for $i=1,2, \ldots, n$ and $t \in[a, b]$. Thus $T y \in C(\vec{H})$; that is, $T(C(\vec{H})) \subset C(\vec{H})$.

If $H \equiv n \max _{1 \leq i \leq n} H_{i}$, then $C(\vec{H}) \subset C(H)$. Moreover, $T(C(\vec{H})) \subset T(C(H))$. Since $T(C(H))$ is relatively compact, $T(C(\vec{H}))$ must also be relatively compact. Since $T$ is continuous on $C(H), T$ is continuous on $C(\vec{H})$. Since $C(\vec{H})$ is a closed, convex subset of $C[a, b]$, we may conclude from Lemma 2.4 that $T$ has a fixed point $x \in C(\vec{H})$; that is, the problem in (1.5), (1.6) has a solution $x \in C(\vec{H})$.

In order to give examples illustrating the improvements obtained in Theorems 3.2 and 3.3, we make the following observation.

LEMMA 3.4. If $\mathbf{L}=\mathbf{L}_{0}$, then (A4) is satisfied for any $A(t)$; that is, the problem

$$
x^{\prime}=A(t) x, \quad \mathbf{L}_{0}(x)=x(a)=r,
$$

has a unique solution on $[a, b]$ for any $A(t)$ and $r$.

Proof. This is a special case of Lemma 2.6.

4. Examples. Below are two examples indicating the use of our main result.

EXAMPLE 4.1. Consider the initial value problem

$$
x^{\prime}=x, \quad x(a)=0, \quad a \geq 0,
$$

that is, consider the problem (1.5), (1.6) with $A(t) \equiv 0, f(t, x)=x, r=0,0 \leq a<b$, and $\mathbf{L}=\mathbf{L}_{0}$. Since $A(t)$ and $f(t, x)$ are clearly continuous, (A1) and (A2) are satisfied. Property (A3) was established by Lemma 2.1. Property (A4) is an immediate consequence of Lemma 3.4.

Since $\psi(t ; 0) \equiv 0$ for problem (4.1), we have

$$
C(H)=\{y \in C[a, b]:\|y\| \leq H\} .
$$

Moreover, it is easily seen that for problem (4.1),

$$
u(t ; r, y)=r+\int_{a}^{t} y(s) d s .
$$

Since $y_{0}(t) \equiv(H / n, H / n, \ldots, H / n) \in C(H)$, we have

$$
M(H) \equiv \sup _{y \in C(H)}\|u(0, y)\| \geq \max _{[a, b]}\left\|\int_{a}^{t} y_{0}(s) d s\right\| \geq(b-a) H .
$$

If $b-a>1$, then condition (3.1) is violated for any choice of $H$. If we define $H(t) \equiv e^{t}$, we have

$$
C(H(t))=\left\{y \in C[a, b]:\|y(t)\| \leq e^{t}, t \in[a, b]\right\} .
$$


It follows from (4.3) that

$$
M(t, H(t)) \equiv \sup _{y \in C(H(t))}\|u(t ; 0, y)\|=\sup _{y \in C(H(t))} \int_{a}^{t}\|y(s)\| d s \leq \int_{a}^{t} e^{s} d s=e^{t-a} \leq H(t) .
$$

Thus condition (3.3) is satisfied for $H(t) \equiv e^{t}$ and existence of a solution to (4.1) in $C(H(t))$ follows from Theorem 3.2.

EXAMPLE 4.2. Consider the initial value problem

$$
x^{\prime}=\left[\begin{array}{ll}
1 & 0 \\
0 & 1
\end{array}\right] x+\left[\begin{array}{c}
x_{2} \\
\alpha
\end{array}\right], \quad x(a)=0,
$$

where $\alpha>0$; that is, consider problem (1.5), (1.6) with $n=2$,

$$
A(t)=\left[\begin{array}{ll}
1 & 0 \\
0 & 1
\end{array}\right], \quad f(t, x)=\left[\begin{array}{c}
x_{2} \\
\alpha
\end{array}\right]
$$

$r=0$, and $\mathbf{L}=\mathbf{L}_{0}$. The properties (A1) through (A4) are satisfied as in the first example. Since $r=0$, we have

$$
C(H)=\{y \in C[a, b]:\|y\| \leq H\} .
$$

It is easily verified that

$$
u(t ; r, y) \equiv r e^{t-a}+\int_{a}^{t} \exp (t-s)\left[\begin{array}{c}
y_{2}(s) \\
\alpha
\end{array}\right] d s
$$

is the unique solution to

$$
x^{\prime}(t)=\left[\begin{array}{ll}
1 & 0 \\
0 & 1
\end{array}\right] x(t)+\left[\begin{array}{c}
y_{2}(t) \\
\alpha
\end{array}\right], \quad \mathbf{L}_{0} x=x(a)=r
$$

Since

$$
\bar{y}(t)=\left[\begin{array}{c}
0 \\
H
\end{array}\right]
$$

is an element of $C(H)$, we have

$$
\begin{aligned}
M(H) & \equiv \sup _{y \in C(H)}\|u(0, y)\| \geq\|u(0, \bar{y})\| \\
& \geq \max _{[a, b]}\left\|\int_{a}^{t} \exp (t-s)\left[\begin{array}{c}
H \\
\alpha
\end{array}\right] d s\right\| \\
& \geq(H+\alpha)(\exp (b-a)-1) .
\end{aligned}
$$

If $b-a>\ln 2$, then $M(H)>H$ for every $H>0$; that is, equation (3.1) is violated for any $H>0$. Let $H_{1} \equiv(\exp (b-a)-1)^{2} \alpha, H_{2} \equiv(\exp (b-a)-1) \alpha$, and $\vec{H} \equiv\left(H_{1}, H_{2}\right)$.

Since $r=0$,

$$
C(\vec{H})=\left\{y \in C[a, b]:\left|y_{1}(t)\right| \leq H_{1},\left|y_{2}(t)\right| \leq H_{2}, t \in[a, b]\right\} .
$$


It follows from (4.10) that

$$
\begin{aligned}
M_{1}(\vec{H}) & \equiv \sup _{y \in C(\vec{H})}\left(\max _{[a, b]}\left|u_{1}(t ; 0, y)\right|\right) \\
& \leq \sup _{y \in C(\vec{H})}\left(\max _{[a, b]}\left|\int_{a}^{t} \exp (t-s) y_{2}(s) d s\right|\right) \\
& \leq \sup _{y \in C(\vec{H})}\left(\max _{[a, b]}\left|y_{2}(s)\right| \int_{a}^{b} \exp (b-s) d s\right) \\
& \leq H_{2}(\exp (b-a)-1)=H_{1}, \\
M_{2}(\vec{H}) & \equiv \sup _{y \in C(\vec{H})}\left(\max _{[a, b]}\left|u_{2}(t ; 0, y)\right|\right) \\
& \leq \sup _{y \in C(\vec{H})}\left(\max _{[a, b]}\left|\int_{a}^{t} \exp (t-s) \alpha d s\right|\right) \\
& \leq \alpha(\exp (b-a)-1)=H_{2} .
\end{aligned}
$$

Thus (3.5) is satisfied and by Theorem 3.3, equation (4.7) must have a solution in $C(\vec{H})$. Let

$$
S(H) \equiv\{y \in C[a, b]:\|y\| \leq H\} .
$$

As an alternative to the mapping $T$ employed in the proof of Theorems 3.1, 3.2, and 3.3, we might have considered the mapping

$$
T_{1}: S(H) \longrightarrow C[a, b]
$$

defined by

$$
T_{1} z \equiv f(t, w(t ; r, z)),
$$

where $w(t ; r, z)=w(r, z)$ is the unique solution to

$$
x^{\prime}=A(t) x+z(t), \quad \mathbf{L} x=r .
$$

Note that if $T_{1} z=z$ for some $z \in S(H)$, then

$$
w^{\prime}(t ; r, z)=A(t) w(t ; r, z)+z(t)=A(t) w(t ; r, z)+f(t, w(t ; r, z))
$$

on $[a, b]$ and $\mathbf{L} w(r, z)=r$; that is, $w(r, z)$ is a solution to (1.5), (1.6).

\section{REFERENCES}

[1] H. A. Antosiewicz, Boundary value problems for nonlinear ordinary differential equations, Pacific J. Math. 17 (1966), 191-197. MR 33\#6003. Zbl 138.32902.

[2] L. E. Bobisud and D. O'Regan, Boundary value problems for first-order differential equations, Proc. Amer. Math. Soc. 99 (1987), no. 3, 501-506. MR 88b:34019. Zbl 615.34012.

[3] N. Dunford and J. T. Schwartz, Linear Operators. I. General Theory, with the assistance of W. G. Bade and R. G. Bartle. Pure and Applied Mathematics, vol. 7, Interscience Publishers, Inc., New York, Interscience Publishers, Ltd., London, 1958. MR $22 \# 8302$. Zbl 084.10402. 
[4] I. P. Gavrilyuk and V. L. Makarov, Representation and approximation of the solution of an initial value problem for a first order differential equation in Banach spaces, Z. Anal. Anwendungen 15 (1996), no. 2, 495-527. MR 97h:65076. Zbl 849.65040.

[5] P. Hartman, Ordinary Differential Equations, John Wiley \& Sons, Inc., New York, London, Sydney, 1964. MR 30\#1270. Zbl 125.32102.

[6] S. Heikkilä and V. Lakshmikantham, On first order differential equations in ordered Banach spaces, Inequalities and Applications, pp. 293-301 (R.-P. Agarwal, ed.), World Sci. Ser. Appl. Anal., vol. 3, World Sci. Publishing, River Edge, NJ, 1994. MR 95g:34095. Zbl 882.34063.

[7] V. Lakshmikantham and S. Leela, Existence and monotone method for periodic solutions of first-order differential equations, J. Math. Anal. Appl. 91 (1983), no. 1, 237-243. MR 84f:34059. Zbl 525.34031.

[8] J. J. Nieto, On the existence of periodic solutions for a class of first order differential equations, Applicable Anal. 20 (1985), no. 1-2, 103-106. MR 87f:34047. Zbl 572.34033.

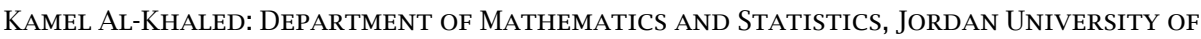
SCIENCE AND TECHNOLOGY, IRBID 22110, JORDAN

E-mail address: app7ied@just.edu.jo 


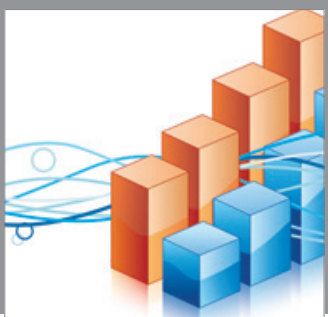

Advances in

Operations Research

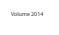

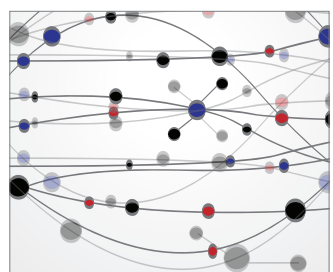

\section{The Scientific} World Journal
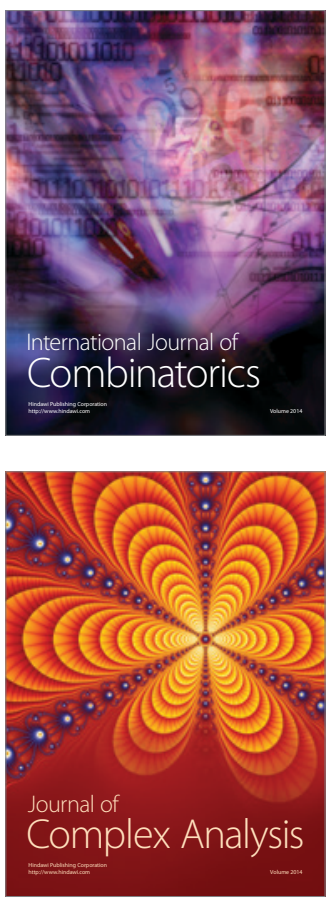

International Journal of

Mathematics and

Mathematical

Sciences
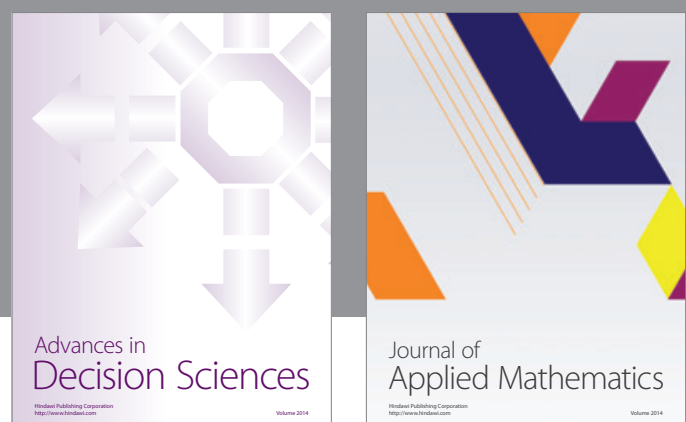

Journal of

Applied Mathematics
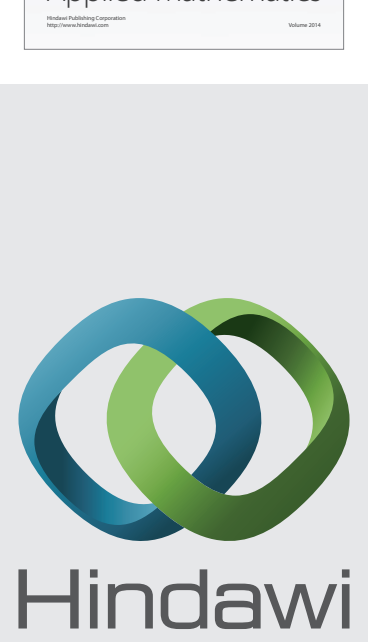

Submit your manuscripts at http://www.hindawi.com
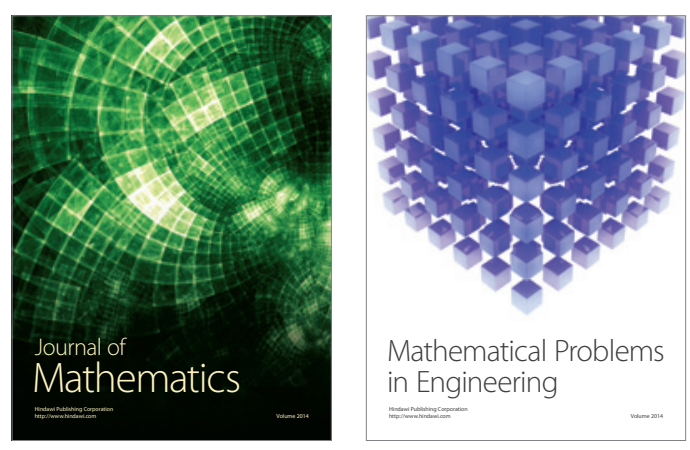

Mathematical Problems in Engineering
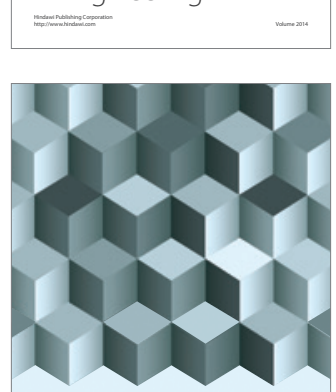

Journal of

Function Spaces
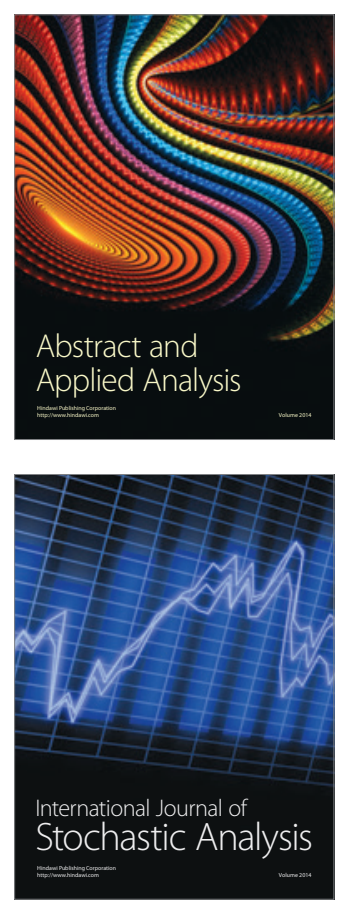

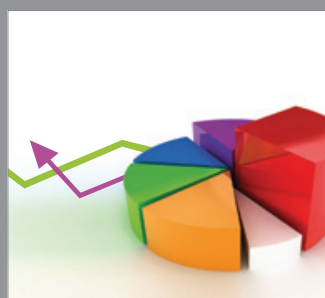

ournal of

Probability and Statistics

Promensencen
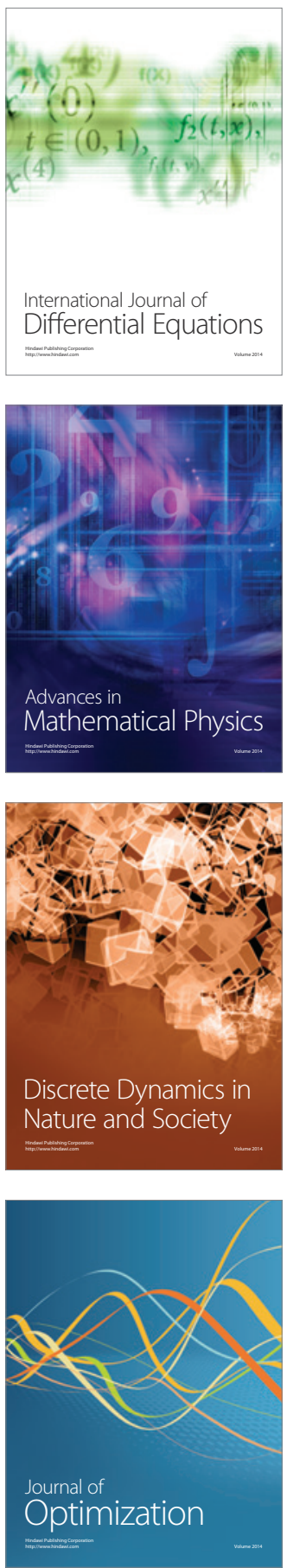